\title{
THE DETERMINATION OF BASAL GLYCEMIA AND GLUCOSE TOLERANCE CURVE: THE MANNERS OF BLOOD SAMPLING
}

\author{
Věroslav Golda \\ Institute of Experimental Neurosurgery, Charles University, Faculty of Medicine and Teaching Hospital, \\ Hradec Králové; (Head: doc. MUDr. J. Náhlovský, CSc.)
}

\begin{abstract}
Summary: There were analyzed the conditions under which the basal glycemia and/or glucose tolerance curve is not contaminated by stress induced changes in glycide metabolism. When the basal glycemia was monitored,blood was sampled to heparinized capillaries from retrobulbar plexus under the light ether anaesthesia or by decapitation without narcosis. The animal represented the control for itself. No differences was found in basal glycemia under the two mentioned blood sampling. In the second series of experiments glycemia was monitored in the time schedule which is used in glucose tolerance test, i.e.,blood sampling was performed 30,60,120 and 180 min after the first sampling from retrobulbar plexus either under light ether anaesthesia (in $14 \mathrm{~h}$ starvated rats or in rats with free access to diet) or under Nembutal anaesthesia (in $14 \mathrm{~h}$ starvated rats). No differences were found in glycemia when two types of narcosis is compared. No signs of augmentation were detected. In the last series when blood sampling was taken in two sec intervals, time dependent augmentation of stress glucose elevation was found. The augmentation was more expressed in the rats with free access to diet than in starvated animals.
\end{abstract}

Key words: Blood sampling; Basal glycemia; GTT; Retrobulbar plexus; Ether anaesthesia; Nembutal anaesthesia; Decapitation

\section{Introduction}

The proper aim of the presented paper is a comparison of different manners of blood sampling when basal glycemia and/or glucose tolerance curve is monitored. The basic problem is represented by restraning of the stress reaction which can take part in the procedure of blood sampling.

Kelsey (2) and Mangili et al. (5) when studying the basal ACTH secretion obtained all basal samples under the ether anaesthesia but in less than $90 \mathrm{~s}$ before a stress-induced increase in ACTH secretion could occured. Sampling was performed by cardiac puncture.

Placing the animal for 3 minutes in an ether vapor saturated area (4) is frequently used as a systemic stressor.On the other hand, Kelsay (2) and Mangili et al (5) used ether narcosis when basal ACTH was monitored. Thus it is apparent that ether must be taken into consideration as stressor only under the some condition, i.e., when there is sufficient time delay between the begining of ether vapor inhalation and the blood sampling used for determination of ACTH and/or plasma corticosterone.

In our recent paper we are comparing basal glycemia when three types of blood sampling were used: two of them were realized under narcosis, i.e., under pentobarbital and/or ether narcosis, and the last one when sampling was realized by decapitation without narcosis.
We have used the schedule where the animal represents the control for itself (see method).

\section{Methods}

\section{Animals}

Experiments under the ether narcosis were performed in the rats of Wistar strain, in the genetically hypertensive obese rats (SHR/N-cp obese) of Koletsky type(3) and in their lean sibling( SHR/N-cp lean). Lean SHR/N-cp rats represent dominant non-obese homozygotes and heterozygotes whereas their obese siblings are recessive homozygotes (cp-cp). The abnormal animals were obtained by Koletsky ( 3 when mating spontaneously hypertensive rat (Okamoto-Aoki strain) with a normotensive Sprague-Dawley male rat. The genetically obese animals appeared after several generations of selective inbreeding of hypertensive off-springs of the original cross.

After weaning at the age of 30 days the animals were kept in groups of four and supplied with water and ST pelleted diet ad libitum. Monitoring was realized in adult rats.

\section{Blood glucose sampling}

Blood was sampled to heparinized capillaries (from retrobulbar plexus under light ether anaesthesia) and after 
about one week delay the second sampling was realized when animals were decapitated without narcosis. Thus, the animal represents the control for itself.

Under the term of light ether anaesthesia we understand the procedure which enable us the blood sampling before the development of stress induced changes in glycemia. The ether narcosis is applied in glass vessel $(10 \times 10 \times 20 \mathrm{~cm})$ where at the bottom is metal grid which is covered by paper-wool. The grid and paper-wool make imposible the direct contact of the animal with liquid ether. For every narcotization cca $3 \mathrm{ml}$ of ether was applied. The glass vessel with the rat is covered by a lid and the behaviour of the animal is observed. When the rat falls asleep (50 - $60 \mathrm{~s}$ after beginning of narcotization), the blood sampling from retrobulbar plexus is performed and finished up to 60 $70 \mathrm{~s}$ after the beginning of narcotization.

Basal glycaemia and glucose tolerance Blood was sampled to heparinized capillaries (from retrobulbar plexus under light ether anaesthsia) before glucose loading (basal glycaemia) as well as 30,60,120 and 180 min after glucose loading. Glucose $(3 \mathrm{~g} / \mathrm{kg}$ b.w., $30 \%$ solution) was applied intragastrically after $14 \mathrm{~h}$ starvation. Glycemia was estimated enzymatically (Oxochrom glucose, Lachema). Glucose tolerance is expressed as a sum of glycemia obtained 30,60,120 and $180 \mathrm{~min}$ after glucose loading (,area under the glucose tolerance curve"). Basal glycemia and glucose tolerance was monitored under Nembutal anaesthesia $(50 \mathrm{mg} / \mathrm{kg}$, i.p.) as well. Basal glycemia without narcosis was obtained when blood sample was taken by decapitation.

\section{Successive blood sampling without glucose loading}

To verify the possible effect of repeated sampling two different types of experiments were performed. In the first one we used identical arrangement which we used in glucose tolerance test, i.e., sampling was realized at time 0,30 , 60,120 and $180 \mathrm{~min}$ in two groups of animals , i.e., after 14 hour starvation and in group without starvation.

The last series of experiments were performed only under the ether narcosis when two minute intervals between individual sampling was used. It was done in the animals after $14 \mathrm{~h}$ starvation and/or in group without starvation. Measurements were carried out in males of Wistar strain.

\section{Results}

When compared basal glycemia obtained under light ether anaesthesia and under Nembutal anaesthesia (sampling in both cases from retrobulbar plexus - see Methods). statistically significant differences were obtained only in one group of rats, i.e., in SHR lean females (Table 1).

The lower level was obtained under decapitation without narcosis.In the mentioned comparison the animal represents the control for itself.

When compared strain differences in the basal glycaemia (Table 1) the lean Koletsky rats show increase in relation to Wistar rats, except glycemia in females where elevation of basal glycemia attained statisticall significance only under the sampling by decapitation without narcosis. Considering the substrain differences in basal glycemia statistical significance was attained only in males when sampling by decapitation without narcosis was used (Table 1).

Table I: Basal glycemia under ether and Nembutal anaesthesia

\begin{tabular}{|l|c|c|c|c|}
\hline Group & $\mathrm{n}$ & $\begin{array}{c}\text { retrobulbar plexus } \\
\mathrm{mmol} / 1\end{array}$ & $\begin{array}{c}\text { decapitation } \\
\mathrm{mmol} / 1\end{array}$ & $\mathrm{P}$ \\
\hline NR M & 8 & $3.36 \pm 0.41$ & $3.02 \pm 0.24$ & n.s. \\
\hline NR F & 8 & $3.62 \pm 0.69$ & $3.71 \pm 0.26$ & n.s. \\
\hline SHR M & 8 & $4.67 \pm 0.86^{\mathrm{D}}$ & $5.17 \pm 0.45^{\mathrm{D}}$ & n.s. \\
\hline SHR F & 8 & $4.10 \pm 0.42$ & $4.59 \pm 0.36^{\mathrm{D}}$ & 0.02 \\
\hline SHR-O M & 10 & $4.13 \pm 1.37$ & $3.49 \pm 0.66^{\mathrm{d}}$ & n.s. \\
\hline SHR-O F & 9 & $4.54 \pm 0.46$ & $4.86 \pm 0.86$ & n.s. \\
\hline
\end{tabular}

Means \pm SD. Statistical significance between basal glycemia when sampling was carried out from retrobulbar plexus under the light ether anaesthsia and/or by decapitation without narcosis was evaluated by parametric paired t-test and controlled by non-parametric Wilcoxon test for matched pairs. Intergroup differences were evaluated by unpaired parametric t-test and controlled by non-parametric MannWitney two sample (non-matched) test. Abbreviations:

SHR - lean Koletsky rats, SHR-O - obese Koletsky rats, M - males,F females, $\mathrm{n}$ - number of rats in group, D - significance of differences between the lean Koletsky rats and the rats of Wistar strain. d - significance of differencers between obese and lean Koletsky rats, D and /or $\mathrm{d}$ : $\mathrm{P}<0.01$.The measure of statistical significance in all cases according to the results in non-parametric test.

Comparing the basal glycaemia under the conditions of repeated sampling (Table 2) of blood at 30,60,120 and 180 min intervals (i.e., in the time schedule which is used in the monitoring glucose tolerance), we have not found any differences under the conditions of Nembutal and/or ether anaesthesia. In both cases the blood was sampled to heparinized capillaries from retrobulbar plexus. When we compare the glycemia under the mentioned time schedule in the starvated and/or unstarvated animals, the elevation shows statistical significance at two intervals in the rats without starvation.

Table 2: Glycaemia - repeated sampling of blood at 30,60, 120 and $180 \mathrm{~min}$ intervals

\begin{tabular}{|l|c|r|r|r|r|r|}
\hline Group & & \multicolumn{5}{|c|}{ Interval in min } \\
& $\mathrm{n}$ & \multicolumn{1}{|c|}{0} & \multicolumn{1}{c|}{30} & 60 & \multicolumn{1}{c|}{120} & \multicolumn{1}{c|}{180} \\
\hline Ether with & 4 & 4.02 & 4.02 & 4.29 & 3.60 & 3.82 \\
starvation & & \pm 0.46 & \pm 0.27 & \pm 0.44 & \pm 0.23 & \pm 0.53 \\
\hline Nembutal with & 8 & 3.86 & 4.10 & 4.15 & 3.66 & 3.26 \\
starvation & & \pm 0.59 & \pm 1.92 & \pm 0.96 & \pm 0.56 & \pm 0.53 \\
\hline Ether without & 4 & 4.59 & 4.93 & 4.70 & 4.82 & 4.45 \\
starvation & & \pm 0.18 & $\pm 0.37 \mathrm{c}$ & \pm 0.37 & $0.17 \mathrm{c}$ & \pm 0.46 \\
\hline
\end{tabular}

Means \pm SD. Presented statistical evaluation of differences in glycemia in individual intervals obtained by sampling under different conditions,i.e., under ether anaesthesia when the animals were with starvation and/or without starvation, and under Nembutal anaesthesia when the animals were with starvation (see Methods). Abbreviations:

$\mathrm{c}-\mathrm{P}<0.02$. Differences were statistically evaluated by parametric t-test and controlled by non-parametric Mann-Witney test. The measure of statistical significance is according to the results obtained in the last mentioned test. 
The factor of starvation is profoundly expressed also under the repeated sampling of blood at two min intervals when the repeated ether anaesthesia was applied (Table 3). There is profound difference not only in the level of basal glycemia (see point 1), but quite different steepness is apparent, being much higher in the group without starvation.When the glycemia at the first sec.is taken as $100 \%$, then the glycemia at 16 th sec represents $153 \%$ (see upper row in Table 3, i,e, under the starvation) and the glycemia at 15 th sec. (see lowe row in Table 3, i.e., under the free access to pellet) represents $193 \%$.

Table 3: Repeated sampling of blood at two min intervals under the repeated ether anaesthesia

\begin{tabular}{|c|c|c|c|c|c|c|c|c|}
\hline 1 & 2 & 4 & 6 & \multicolumn{1}{c|}{8} & 10 & \multicolumn{1}{c|}{12} & \multicolumn{1}{c|}{14} & \multicolumn{1}{c|}{16} \\
\hline 4.24 & 4.29 & 5.01 & 4.73 & 5.25 & 5.75 & 5.78 & 5.65 & 6.48 \\
\pm 0.66 & \pm 0.54 & \pm 0.92 & \pm 0.70 & \pm 1.18 & \pm 0.87 & \pm 0.93 & \pm 1.04 & \pm 1.34 \\
\hline 1 & 3 & 5 & 7 & 9 & 11 & 13 & 15 & \\
\hline 5.01 & 5.62 & 6.46 & 7.15 & 7.95 & 8.37 & 8.79 & 9.67 & \\
\hline $\pm 0.21^{\mathrm{b}}$ & $\pm 0.41^{\mathrm{c}}$ & \pm 0.57 & $\pm 0.25^{\mathrm{c}}$ & $\pm 0.29^{\mathrm{c}}$ & $\pm 0.47^{\mathrm{c}}$ & $\pm 0.51^{\mathrm{c}}$ & $\pm 0.27^{\mathrm{c}}$ & \\
\hline
\end{tabular}

Table 3: Means \pm SD. The top row: animals under the 14 h starvation $(n=4)$, the botton row: animals without starvation with pellet diet ad libitum $(n=4)$ The level of glycaemia at individual steps of experiments (1st versus 1st min, 2nd min versus 3rd min, 4th min versus 5 th min ect).

Abbreviations: $\mathrm{b}-\mathrm{P}<0.05, \mathrm{c}-\mathrm{P}<0.02, \mathrm{~d}-\mathrm{P}<0.01$.

Differences were statistically evaluated by parametric t-test and controlled by non-parametric Mann-Witney test.The measure of statistical sigificance in all cases according to the results in non-parametric test.

\section{Discussion}

It is apparent that basal glycemia obtained by sampling under the ether anaesthesia and the sampling by decapitation without narcosis is comparable.Moreover, our data obtained in normotensive rats of Wistar strain are comparable with the results presented by Hefco (1). This author studied the basal glycemia in the males of Wistar as well. He removed the blood samples from tail veins. No more details are presented by the mentioned author. He described three experiments with the following basal glycemia levels:

$4.18 \pm 0,22(n=7), 1,3.41 \pm 0.17 \quad(n=10)$, and $3.80 \pm 0.06$ $(\mathrm{n}=7) \mathrm{mmol} / \mathrm{l}$ (data were presented in $\mathrm{mg} \%$ ). The presented data suggest that intergroup differences can represented about $0.7 \mathrm{mmol} / 1$.

Now to the repeated narcotization with ether.

Our data suggest that repeated application of ether shows the stress effect (in our case the elevation of glycemia) in dependance on the frequency of an exposition the animal to ether vapor. When the intersampling intervals are $30 \mathrm{sec}$ or more,we can see that the level of glycemia is aqual or near to basal glycemia (when sampling was made under the condition of starvation) or to the first sample (when sampling was made in the animal with free access to pellets (see Table 2). No augmentation in stress reaction can be found. No differences were found when sampling was per- formed under ether and/or Nembutal anaethesia (Table 2, the upper two rows. As to the pentobarbital anaesthesia (see in our experiments Nembutal), Moss (6) found that this type of narcotic did not alter any ACTH levels.

On the other hand, when the intersampling intervals are $2 \mathrm{sec}$, then there can be observed profound augmentation of stress reaction in the form of elevated glycemia (see Table 3). But it is apparent that the augmentation of stress reaction is highly state dependent,i.e., the augmentation is twofold higher in the animals which were not exposed to starvation. Where is a proper reason of the mentioned differences it is not clear. It cannot be a priori excluded that the different state of available glycogen depots can be judged as a main intervening variable.

\section{Acknowledgement}

This paper was supported By Internal Grant Agency of Ministry of Health of the Czecch Republic No 3684-3. The authors wish to thank Carl T. Hansen, Animal Genetics Division, National Institute of Health, Bethesda, USA, for providing the genetically hypertensive rats of Koletsky type.

\section{References}

1. Hefco VP. The role of the medial hypothalamus nervous afferents in inducing hyperglycaemia in rats by certain stressors. Rev Roum Endocrinol 1972;9:97-102.

2. Kelsay JE. Role of pituitary-adrenocortical system in mediating avoidance behavior of rats with septal lesions. J Comp Physiol Psychol 1975;88:271-80.

3. Koletsky S. Pathologic findings and laboratory data in a new strain of hypertensive rats. Am J Pathol 1975;80:11940.

4. Květňanský R, Dobrakovová M, Ježová D, Opešálová Z, Lichardus B, Makara G. Hypothalamic regulation of plasma catecholamine levels during stress: effect of vasopressin and CRF. In: Stress: Neurochemical and Humoral Mechanisms. GR van Loon, R.Květňanský, R.McCarty, J Axelrod (Eds), New York:Gordon and Breach Science Publishers, 1989:549-70.

5. Mangili G, Motta M, Martini L. Control of adrenocorticorticotropic hormone secretion. Cit. Kelsey JE J Comp Physiol Psychol 1975;88:271-80.

6. Moss IR, Inman JG, Porter JC, Faucher DJ. Ontogeny of plasma, CSF and brainstem ACTH in piglets: effects of hypoxia and anaesthesia. Neuroendocrinology 1990;51:586-91.

Submitted April 1998.

Accepted May 1998.

Doc. MUDr. PhDr. Věroslav Golda, CSc., Institute of Experimental Neurosurgery, Charles University, Faculty of Medicine and Teaching Hospital, 50005 Hradec Králové, Czech Republic. 